\title{
Preparation and Cyclodextrin Solubilization of the Antibacterial Agent Benzoyl Metronidazole
}

\author{
Qing Huang, Benpeng Li, Shuo Yang, Peipei Ma, and Zhizhong Wang \\ School of Pharmacy, Ningxia Medical University, Yinchuan, Ningxia 750004, China \\ Correspondence should be addressed to Zhizhong Wang; zhizhongwang1976@163.com
}

Received 24 April 2013; Accepted 25 June 2013

Academic Editors: A. Sacchetti, A. K. Saikia, and A. Sirit

Copyright (C) 2013 Qing Huang et al. This is an open access article distributed under the Creative Commons Attribution License, which permits unrestricted use, distribution, and reproduction in any medium, provided the original work is properly cited.

\begin{abstract}
A one-pot method for the preparation of benzoyl metronidazole was achieved by using $N, N^{\prime}$-carbonyldiimidazole as a coupling reagent. Moreover, it was found that the byproduct imidazole as the catalyst promoted the reaction. In addition, the $\beta$-cyclodextrin solubilization of benzoyl metronidazole was investigated by phase-solubility method. The phase-solubility studies indicated that the solubility of benzoyl metronidazole $(S=0.1435 \mathrm{~g} / \mathrm{L})$ was substantially increased 9.7 -fold $\left(S^{\prime}=1.3881 \mathrm{~g} / \mathrm{L}\right)$ by formation of $1: 1$ benzoyl metronidazole/ $\beta$-cyclodextrin complexes in water, and the association constant $K_{a}$ value was determined to be 251 $( \pm 23) \mathrm{dm}^{3} /$ mol. Therefore, $\beta$-cyclodextrin can work as a pharmaceutical solubilizer for benzoyl metronidazole and may improve its oral bioavailability.
\end{abstract}

\section{Introduction}

Metronidazole (MTZ), 2-(2-methyl-5-nitro-1H-imidazol-1yl)ethanol, is known to be a powerful antiprotozoal and antibacterial drug. It is clinically effective in trichomoniasis, amoebic colitis, and giardiasis [1]. However, it has a bitter taste and is not acceptable to some young patients. Benzoyl metronidazole, the benzoyl ester of metronidazole, is tasteless and has also been widely used because of its greater palatability [2]. In addition, formulation of benzoyl metronidazole in aqueous drug formulation has been hampered by its low aqueous solubility.

The conventional routes for synthesis of benzoyl metronidazole were the two-step synthesis as shown in Scheme 1, which required the preparation of benzoyl chloride; the reaction required strict anhydrous conditions $[3,4]$, and the reactor should be corrosion resistant. In addition, in the combination of benzoyl chloride and metronidazole, the deacid reagent such as pyridine was needed to promote the reaction. $N, N^{\prime}$-carbonyldiimidazole (CDI) is one of several commonly used reagents for activating carboxyl groups. It is relatively cheap, and the only byproducts are carbon dioxide and imidazole which, being relatively benign, are unlikely to cause problems on scale up [5]. Herein, we report an improved procedure for the preparation of benzoyl metronidazole in one pot by using $N, N^{\prime}$-carbonyldiimidazole as a coupling reagent, and the byproduct imidazole as the catalyst can promote the reaction (Scheme 2). In addition, the use of $\beta$-cyclodextrin $(\beta-\mathrm{CD})$ to increase the water solubility of benzoyl metronidazole was described.

\section{Materials and Methods}

2.1. Materials and Instruments. All the experiments were performed with analytical-grade chemicals and solvents. $\beta-C D$ was recrystallized twice from distilled water and dried under reduced pressure at $110^{\circ} \mathrm{C}$ for $24 \mathrm{~h}$ before use. Dichloromethane (DCM) was dried by $\mathrm{CaCl}_{2}$ for $12 \mathrm{~h}$ and distilled prior to use. The progresses of the reactions were monitored by TLC on $0.25 \mathrm{mM}$ thick layers of silica gel $\mathrm{GF}_{254}$ developed with solvent system, AcOEt : petroleum ether (1:1, v/v). ${ }^{1} \mathrm{H}$ NMR spectra were recorded on Bruker 400 spectrometer in $\mathrm{CDCl}_{3}$ solutions with TMS (tetramethylsilane) as standard. The melting point determinations were carried out on a XRC-I melting point apparatus. The spectroscopic measurements were performed on a double-beam UV-vis spectrophotometer (Shimadzu UV-2550). 


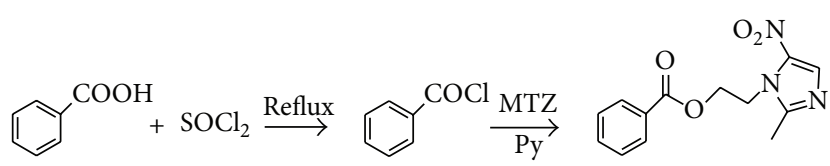

SCHEME 1

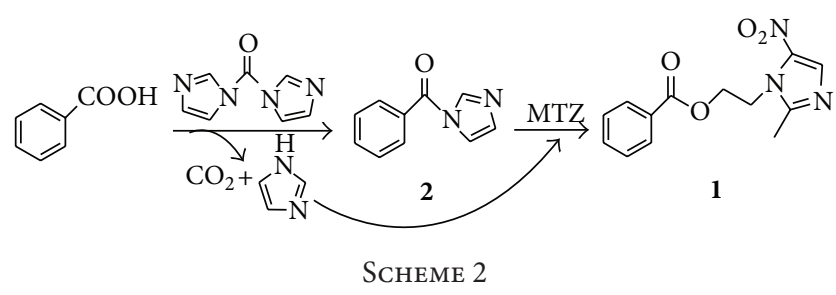

2.2. Facile Preparation of Benzoyl Metronidazole. To a solution of benzoic acid $(1.0 \mathrm{~g}, 8.2 \mathrm{mmol})$ in $40 \mathrm{~cm}^{3}$ anhydrous DCM, $N, N^{\prime}$-carbonyldiimidazole $(1.6 \mathrm{~g}, 9.8 \mathrm{mmol}$ ) was added at room temperature. After being stirred for $4 \mathrm{~h}$, metronidazole $(1.4 \mathrm{~g}, 8.2 \mathrm{mmol})$ in $100 \mathrm{~cm}^{3}$ anhydrous DCM was added. Then the resulting solution was refluxed for $10 \mathrm{~h}$ and concentrated. The residue was dissolved in $80 \mathrm{~cm}^{3} \mathrm{DCM}$ and sequently washed with $1 \mathrm{M}$ sodium carbonate solution $\left(2 \times 20 \mathrm{~cm}^{3}\right), 10 \% \mathrm{HCl}\left(2 \times 15 \mathrm{~cm}^{3}\right)$, and distilled water $\left(3 \times 20 \mathrm{~cm}^{3}\right)$. The organic layer was separated, dried over anhydrous sodium sulphate, and concentrated in vacuo. Finally, the concentrate was crystallized from ethanol to give 1 (1.97 g). Yield 87\%; M.p.: 99.3-100.4 ${ }^{\circ} \mathrm{C}\left([6] 99-102^{\circ} \mathrm{C}\right)$. ${ }^{1} \mathrm{H}$ NMR $\left(400 \mathrm{MHz}, \mathrm{CDCl}_{3}\right): \delta 2.49\left(\mathrm{~s}, 3 \mathrm{H}, \mathrm{CH}_{3}\right.$ group, imidazole), 4.69 (t, 2H, N-CH ${ }_{2}$ ), 4.72 (t, 2H, O- $\mathrm{CH}_{2}$ ), 7.427.93 (m, 5H, Ar-H), and 7.98 (s, 1H, imidazole ring $\mathrm{H})$.

2.3. Solubility Measurements. Phase-solubility studies of benzoyl metronidazole in aqueous solutions of $\beta$-CD were carried out according to the Higuchi-Connors procedure [7]. Various amounts of $\beta$-CD were generally dissolved in distilled water, and excess amounts of benzoyl metronidazole were loaded in glass vials. The vials were shaken in a temperature-controlled room at $298 \mathrm{~K}$ for $24 \mathrm{~h}$ to achieve the equilibrium. Appropriate aliquots were then withdrawn and filtered appropriately diluted with distilled water, and the total concentration of benzoyl metronidazole in the filtrate was analyzed by UV-vis absorbance spectrum. The absorbances at $316 \mathrm{~nm}$ for benzoyl metronidazole were measured, in order to determine the concentration of the dissolved benzoyl metronidazole.

\section{Results and Discussion}

3.1. Facile Preparation of Benzoyl Metronidazole. In our work, we found that simply mixing benzoyl imidazole (2) with metronidazole in DCM did not result in an obvious reaction in $24 \mathrm{~h}$. Imidazole can efficiently promote the reaction. Furthermore, the stronger bases such as $\mathrm{Na}_{2} \mathrm{CO}_{3}$ or $\mathrm{Et}_{3} \mathrm{~N}$ did not distinctly enhance the reaction rate.

$N, N^{\prime}$-carbonyldiimidazole is a useful, general carboxylic acid activating reagent, and its byproduct imidazole can serve as the catalyst. Therefore, benzoyl metronidazole (1) was prepared in a simple, two-step sequence in just one reactor. In the first step, based on TLC analysis, the reaction between benzoic acid and $N, N^{\prime}$-carbonyldiimidazole was quantitative within $4 \mathrm{~h}$ at room temperature. In the second step, the coupling of metronidazole to the benzoyl group was accomplished in $10 \mathrm{~h}$ under reflux in DCM.

The optimized conditions for the synthesis of benzoyl metronidazole are benzoic acid/N, $N^{\prime}$-carbonyldiimidazole $/$ metronidazole $=1 / 1.2 / 1(\mathrm{~mol} / \mathrm{mol})$. The purity and structure were confirmed by NMR, TLC, and melting point.

3.2. Solubilization of Benzoyl Metronidazole by $\beta$-CD. Benzoyl metronidazole possesses poor solubility in water $(S=$ $\left.0.1435 \mathrm{~g} / \mathrm{L}, 5.22 \times 10^{-4} \mathrm{M}\right)$. Solubilization of poorly soluble drugs is one of the most important physicochemical properties for drug development since more than one-third of drugs are poorly water soluble or water insoluble [8]. In order to prepare a liquid dosage formulation of these drugs, a solubilization technique is usually applied. $\beta$-CD has been widely used as an excipient in the pharmaceutical industry for improving some properties of drugs, such as solubility, stability, absorption, and/or bioavailability, by forming the inclusion complexes [9]. In our work, the $\beta$-CD solubilization of benzoyl metronidazole in purified water was first assessed. The phase-solubility studies indicated that $\beta$-CD increased the aqueous solubility of benzoyl metronidazole by approximately 9.7-fold $\left(S^{\prime}=1.3881 \mathrm{~g} / \mathrm{L}, 5.05 \times 10^{-3} \mathrm{M}\right)$.

The decreasing of the UV-vis absorbance intensity and the blue shifted absorbance maximum suggest the formation of an inclusion complex between benzoyl metronidazole and $\beta$ $\mathrm{CD}$ (Figure 1). The linearity in the plot revealed the formation of 1:1 complex between benzoyl metronidazole and $\beta$-CD (Figure 2 ). The stability constant $K_{a}$ can be determined by using Benesi-Hildebrand equation [10]:

$$
\frac{1}{\Delta A}=\frac{1}{\Delta \varepsilon[S]}+\frac{1}{\Delta \varepsilon K_{a}[S][C]},
$$

where $[S]$ and $[C]$ represent the concentrations $\left(\mathrm{mol} / \mathrm{dm}^{3}\right)$ of substrate and $\beta$-CD, respectively. $\Delta A$ is the change in the absorbance of the substrates before and after addition of $\beta$-CD, and $\Delta \varepsilon$ is the difference in the molar absorptivities between complexed and free substrate. Plotting $1 / \Delta A$ against $1 /[C]$ gives a straight line with slope equal to $1 / \Delta \varepsilon K_{a}[S]$. The association constant $K_{a}$ was directly obtained from the intercept/slope ratio. The $K_{a}$ value was determined to be $251( \pm 23) \mathrm{dm}^{3} / \mathrm{mol}$ for the inclusion complexation of $\beta-\mathrm{CD}$ with benzoyl metronidazole.

\section{Conclusions}

An improved method for the preparation of benzoyl metronidazole via a one-pot reaction was developed by using $N, N^{\prime}$-carbonyldiimidazole as a coupling reagent, which does not require extra catalysts. Compared with routine synthetic methods, these procedures may become an efficient route for the synthesis of benzoyl metronidazole. In addition, the phase-solubility studies indicated that the solubility of 


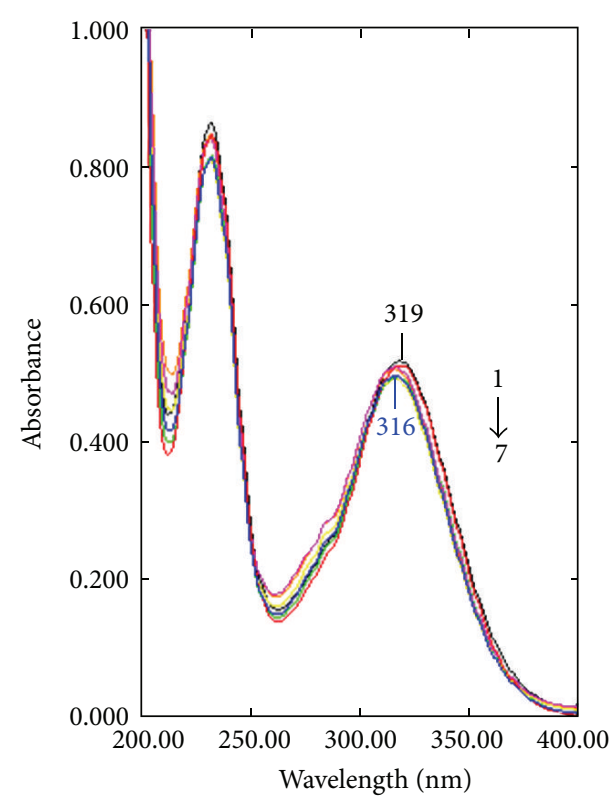

FIGURE 1: UV-vis absorption spectra of benzoyl metronidazole $(5.7 \times$ $10^{-5} \mathrm{M}$ ) at various concentrations of $\beta-\mathrm{CD}$ at $298 \mathrm{~K}$ : (1) no $\beta$-CD, (2) $1.6 \mathrm{mM}$, (3) $3.2 \mathrm{mM}$, (4) $4.8 \mathrm{mM}$, (5) $8.0 \mathrm{mM}$, (6) $11.2 \mathrm{mM}$, and (7) $14.4 \mathrm{mM} \beta$-CD.

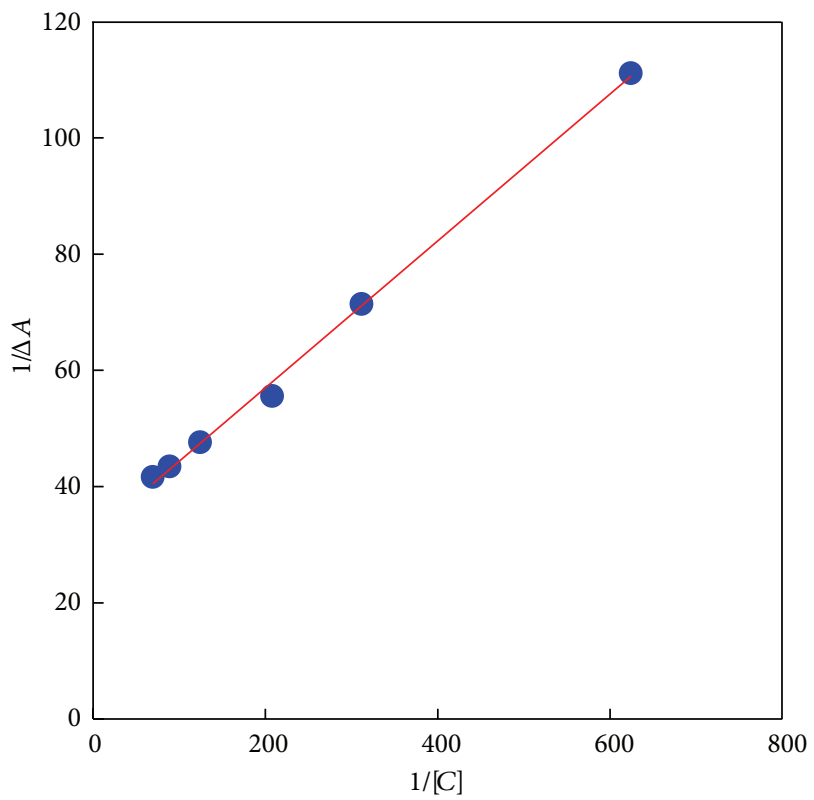

Figure 2: Benesi-Hildebrand plot for the complexation of benzoyl metronidazole with $\beta$-CD.

benzoyl metronidazole $(S=0.1435 \mathrm{~g} / \mathrm{L}$ ) was substantially increased 9.7-fold $\left(S^{\prime}=1.3881 \mathrm{~g} / \mathrm{L}\right)$ by formation of $1: 1$ benzoyl metronidazole/ $\beta$-CD complexes in water, and the association constant $K_{a}$ value was determined to be $251( \pm 23) \mathrm{dm}^{3} / \mathrm{mol}$. Therefore, $\beta$-CD can work as a pharmaceutical solubilizer for benzoyl metronidazole and may improve its oral bioavailability.

\section{Authors' Contribution}

Qing Huang and Benpeng Li contributed equally to this work and are joint first authors.

\section{Acknowledgments}

Undergraduate Innovation Project and Scientific Research Foundation of the Higher Education Institution of Ningxia (no. NGY2012071) are gratefully acknowledged.

\section{References}

[1] T. J. Mehta, S. S. Rajput, M. R. Patel, K. R. Patel, N. M. Patel, and M. Mothilal, "Formulation, development and optimization of metronidazole compression coated tablets," Der Pharmacia Lettre, vol. 3, no. 5, pp. 94-103, 2011.

[2] N. T. Vu, V. Aloumanis, M. J. Ben et al., "Stability of metronidazole benzoate in SyrSpend SF one-step suspension system," International Journal of Pharmaceutical Compounding, vol. 12, no. 6, pp. 558-564, 2008.

[3] S. Dubey, V. Jain, and G. B. Precthi, "Evaluation of lipophilicity, antimicrobial activity and mutagenicity of some novel ester prodrugs of metronidazole," Indian Journal of Chemistry B, vol. 48, no. 11, pp. 1571-1576, 2009.

[4] M. Johansen and C. Larsen, "A comparison of the chemical stability and the enzymatic hydrolysis of a series of aliphatic and aromatic ester derivatives of metronidazole," International Journal of Pharmaceutics, vol. 26, no. 3, pp. 227-241, 1985.

[5] E. K. Woodman, J. G. K. Chaffey, P. A. Hopes, D. R. J. Hose, and J. P. Gilday, "N,N'-carbonyldiimidazole-mediated amide coupling: significant rate enhancement achieved by acid catalysis with imidazole•HCI," Organic Process Research and Development, vol. 13, no. 1, pp. 106-113, 2009.

[6] EDQM, EUROPEAN PHARMACOPOEIA 7. 0, metronidazole benzoate, 01/2008:0934.

[7] T. A. Higuchi and K. A. Connors, "Phase-solubility techniques," Advances in Analytical Chemistry and Instrumentation, vol. 4, pp. 117-122, 1965.

[8] S. Göktürk, E. Çalışkan, R. Y. Talman, and U. Var, "A study on solubilization of poorly soluble drugs by cyclodextrins and micelles: complexation and binding characteristics of sulfamethoxazole and trimethoprim," The Scientific World Journal, vol. 2012, Article ID 718791, 12 pages, 2012.

[9] T. Loftsson and D. Duchêne, "Cyclodextrins and their pharmaceutical applications," International Journal of Pharmaceutics, vol. 329, no. 1-2, pp. 1-11, 2007.

[10] Q.-X. Guo, H.-Y. Liu, X.-Q. Ruan, X.-Q. Zheng, Y.-Y. Shi, and Y.-C. Liu, "Experimental and theoretical studies on the inclusion complexation of $\beta$-cyclodextrin with phenothiazine derivatives," Journal of Inclusion Phenomena, vol. 35, no. 3, pp. 487-496, 1999. 

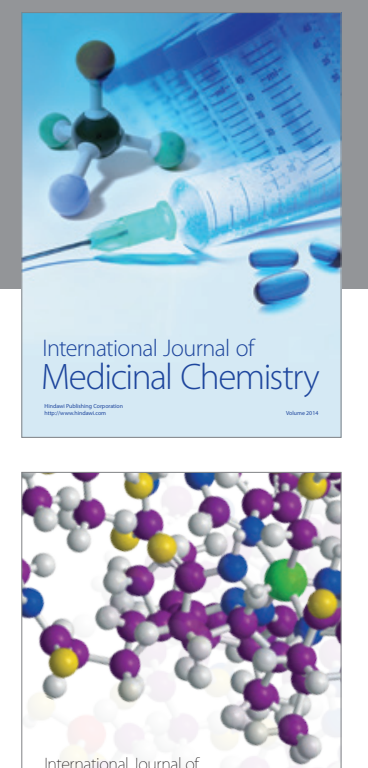

\section{Carbohydrate} Chemistry

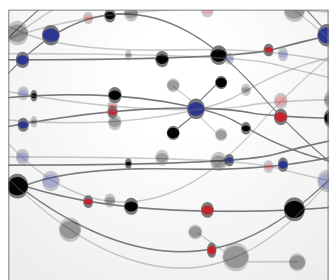

The Scientific World Journal
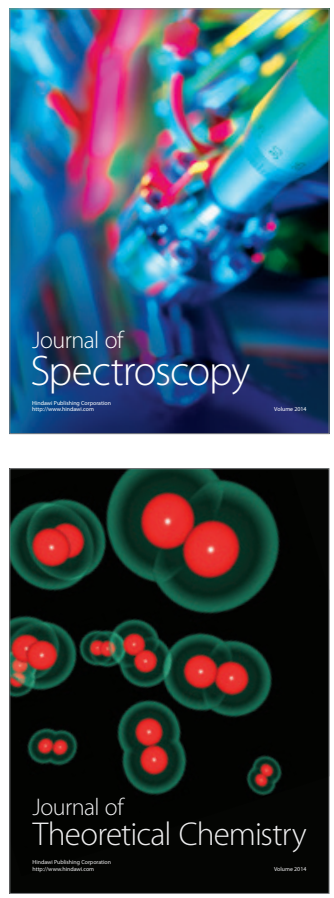
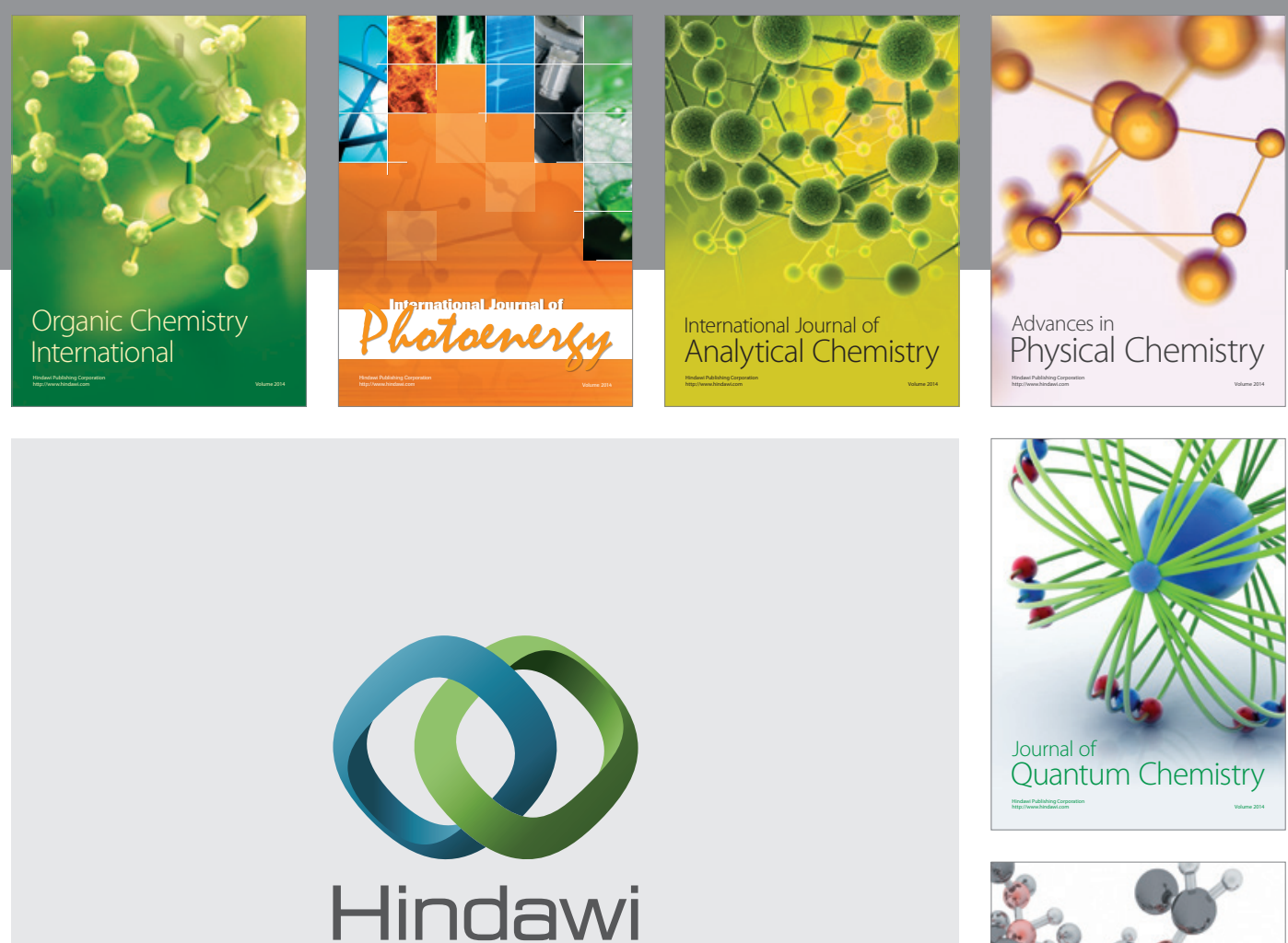

Submit your manuscripts at

http://www.hindawi.com

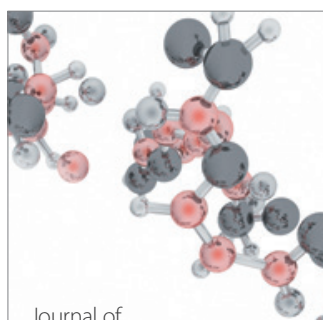

Analytical Methods

in Chemistry

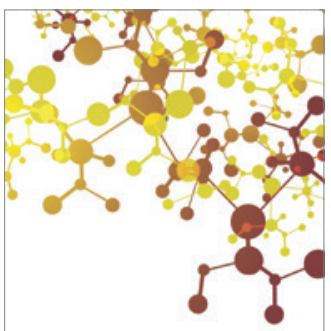

Journal of

Applied Chemistry

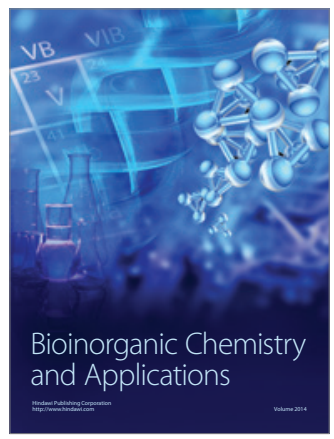

Inorganic Chemistry
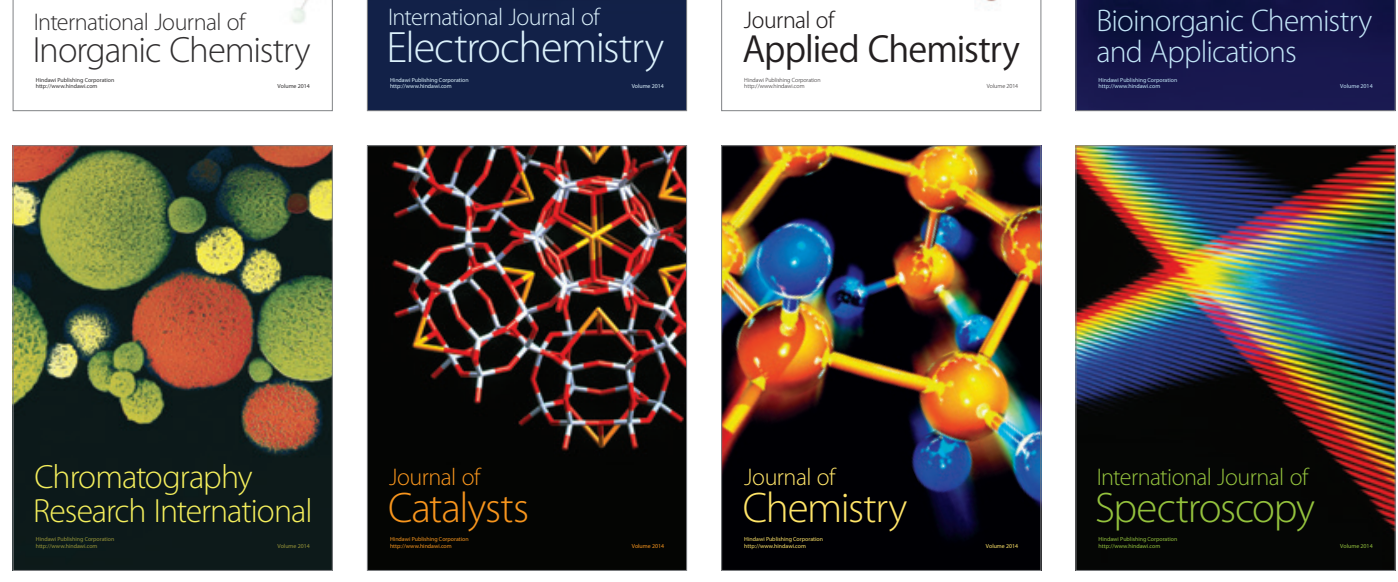